\title{
Getting Good Results From Your Results Section
}

\author{
Abbey Thomas, PhD, ATC ${ }^{1}$, and Jeffrey B. Driban, PhD, ATC, CSCS $^{2}$ \\ ${ }^{1}$ University of North Carolina at Charlotte; ${ }^{2}$ Tufts Medical Center
}

\section{"To simplify complications is the first essential of success." - George Earle Buckle}

The ultimate goal of clinical research is to improve human health. To do this, clinical scientists use the scientific method to generate and refine evidence to help inform clinical decisions. This goal can only be achieved if clinicians can readily access and consume new research findings that may be legitimate and applicable to their patient. Therefore, it is imperative that clinical scientists communicate in a meaningful way to their target audience. Clinical scientists are expected to use the most relevant methods, the most valid and reliable outcome measures, and the optimal statistical analyses in their generation of evidence. However, none of these expectations matter if they fail to clearly explain a study's findings to their most important audience, the clinicians.

Many writers have described the benefits of using the fewest and simplest words needed to describe something complex to an audience. In government, the Plain Language Act ensures that all relevant governmental communications to the public are written in a readily consumable way. Should the clinicians who serve the public expect anything less? In the context of a results section, using plain language means choosing words that allow the clinician to find the information they need, understand that information, and use that information to guide their clinical decision making.

The results section is a critical section where the authors objectively report the evidence in a logical order to support their take-home messages. When considering how to craft your results section, it is helpful to know the journal's target audience and then tailor the results for them. For example, the International Journal of Athletic Therapy \& Training (IJATT) is the professional journal for athletic trainers and athletic therapists. Hence, manuscripts submitted to IJATT should be tailored to clinicians and the results and their interpretation should focus on application to clinical practice. Our goal for this editorial is to offer suggestions on how the results section can be improved to successfully and succinctly deliver a purposeful message to a target audience. See the Callout Box for examples of the points that follow.
Thomas is with the Department of Kinesiology, University of North Carolina at Charlotte, Charlotte, NC, USA. Driban is with the Division of Rheumatology, Allergy, \& Immunology, Tufts Medical Center, Boston, MA, USA. Thomas (afenwick@uncc.edu) is corresponding author.

\section{Callout Box}

Example 1:

- Results with excessive jargon: "Based on the results of the group-by-time repeated-measures ANOVA, there was a statistically significant group-by-time interaction $\left(F_{2,34}=\right.$ 14.56, $p=.002$; Table 1). The post hoc analysis revealed that CAI patients (mean: $36.3 \pm 4.3 \mathrm{~cm}$ ) walked further than healthy controls (mean: $35.2 \pm 3.7 \mathrm{~cm}$ ) during the walking task. There was also a significant main effect for time. Both groups demonstrated increased walking distance at posttest $(36.1 \pm 3.9 \mathrm{~cm})$ compared to pretest $\left(31.2 \pm 4.1 \mathrm{~cm} ; F_{1,24}=\right.$ $13.65, p=.003) . "$

- Results directed toward a clinical audience: "All results for the three tasks are presented in Table 1. Both groups demonstrated a statistically significant increase in walking distance from pretest to posttest $(p=.003)$. The CAI group had a larger increase compared to the control group $(p=.002)$. However, none of the changes exceeded minimum detectable change of $1 \mathrm{~m} . "$

Example 2:

- Results with excessive jargon: "Based on results from four Kruskal-Wallis tests, ROIs with BMLs had higher aBV/TV (small: $p=.0009$; large: $p=.002$ ), aTb.N (small: $p=.005$; large: $p=.004$ ), and aTb.Th (small: $p<.0001$; large: $p=.002$ ) than ROIs without BMLs.

- Results directed toward a clinical audience: "Areas of bone with a bone marrow lesion had more trabeculae (greater number and greater thickness; more bone volume fraction) than areas without a bone marrow lesion (Table 1)."

Example 3:

- Results with excessive jargon: "Results of binary logistic regression analyses revealed statistically significant odds ratios for prediction of free throw shooting percentage for elbow flexion $\mathrm{ROM}(\mathrm{OR}=1.95, p=.002)$ and humerus length $(\mathrm{OR}=2.76, p=.001)$. The combination of elbow flexion ROM and humerus length was also statistically significant $(\mathrm{OR}=1.70, p=.003)$.

- Results directed toward a clinical audience: "We report the relationship between upper extremity characteristics and free throw shooting percentage in Table 1 using odds ratios and $95 \%$ confidence intervals. Basketball players who have a longer humerus, greater elbow flexion ROM, or both have higher free throw shooting percentages than their peers (Table 1)." 


\section{Statistical Significance Versus Clinical Meaningfulness}

Often, the statistical findings represent the hallmark of the results section. Authors set an a priori level of significance for their studies and then conduct complex statistical analyses to see if their findings exceed a critical value based on their a priori decisions. In reality, the $p$ value simply indicates the probability that the observed group distributions occurred by chance. In other words, when comparing groups, a $p$ value represents whether the decisions the authors made to manipulate groups (independent variables) were responsible for the group distributions in the results (dependent variables). The $p$ value tells us nothing about the magnitude of the differences found. It simply represents the likelihood that group distributions occurred "independent" of chance. ${ }^{1}$

\section{Reporting Meaningfulness is Different From Statistical Significance}

While there are many ways to analyze data, statistical significance is often touted to be the most accepted method for making inferences about a study sample back to a target population. There is a growing consensus that this is actually an incomplete (if not incorrect) way to approach reporting results. ${ }^{2}$ Instead of only focusing on statistical significance we should also offer an interpretation that puts the findings in terms of clinical meaningfulness. Traditional statistical analyses report differences in group trends (means and variance). Measures of clinical meaningfulness (e.g., patient-acceptable symptom state, minimally-clinically important difference, minimally-detectable difference) are intended to identify individuals who are most likely to experience the outcome of interest. Hence, pairing statistical significance with clinical meaningfulness can help readers understand that not only is the group, on average, likely different, but also understand in each group how many people might have a good or bad outcome.

\section{Keep it Simple...}

The results section should offer objective evidence to justify the take-home messages in the discussion. For example, people with chronic ankle instability walk slower than healthy individuals. What would the reader expect to see in the results to support this take-home message? In other words, what evidence do the authors need to provide to help the reader to come to the same judgment? To build a case for the take-home message, a table or figure can be worth 1,000 words if it helps a reader quickly understand the results. First, the authors can help simplify the text toward plain language. Second, the authors can provide the numbers in a format where a reader can more easily interpret or compare them. Finally, tables or figures allow the authors to describe the results in different ways in the text and in the table or figure, which may help clarify the findings. For example, rather than stating "the chronic ankle instability (CAI) group walked at an average speed of $2.5 \mathrm{mph}$ whereas the healthy group walked at $3.3 \mathrm{mph}$ " in the text, the authors can state the percent difference between the group averages (e.g., "on average the CAI group walked 33\% slower than the healthy group. [See table for actual differences.]"). Table 1 provides other suggestions to improve the results section after the outcomes and statistics are moved to tables or figures. These tips help simplify the results and deliver clearer take-home messages using terminology commonly used in clinical practice.

\section{Designing an Informative Table}

We noted above the benefit of moving statistics and numeric results out of the text, but not every table effectively delivers the results. The goal is for a table to tell a story without the paper and serve as a quick reference. Hence, we need to ensure the title tells a reader what is in the table or what is the key take-home message. The table also needs to clearly define abbreviations and symbols. The table can also provide an important opportunity to provide details that may make the text challenging to read (e.g., central tendency, variance, test statistics, \% responders). The first table of a paper can offer a detailed description of the study sample with enough detail to help readers figure out if the study sample is like their patient population. Furthermore, the first table can offer a chance to compare the study sample to the larger population from which it was selected (when applicable). In the subsequent tables, the outcomes and treatment arms/groups should typically be the columns. In contrast, predictors, exposures, or factors being compared between groups typically go in rows. In other words, similar elements should read down and not across a table. ${ }^{3}$ Table 2 demonstrates how not to present data in a table while Table 3 offers an example of a nice table that illustrates key elements to contribute to a successful table.

Table 1 Tips and Examples to Improve the Results Section

\begin{tabular}{|c|c|c|}
\hline Tips to Keep the Text Simple & $\begin{array}{l}\text { Ambiguously Written as it Relates to a } \\
\text { Proposition }\end{array}$ & $\begin{array}{l}\text { Plain Language With Clear Support } \\
\text { for a Proposition }\end{array}$ \\
\hline $\begin{array}{l}\text { Avoid making the statistical test the focus of the } \\
\text { sentence }\end{array}$ & $\begin{array}{l}\text { Using an analysis of variance, we found statistically } \\
\text { significant differences in walking speed across groups } \\
\ldots\end{array}$ & $\begin{array}{l}\text { The three groups differed in walking speed, } \\
\text { with group A walking } 33 \% \text { faster than the } \\
\text { other two groups (see Table). }\end{array}$ \\
\hline State the direction of the result & Age was related to walking speed. & $\begin{array}{l}\text { Older adults were more likely to walk } \\
\text { slower. }\end{array}$ \\
\hline $\begin{array}{l}\text { Focus on the construct, not the measurement } \\
\text { (avoid research jargon; except when multiple } \\
\text { outcome measures address one construct) }\end{array}$ & Group A had greater CES-D scores than group B. & $\begin{array}{l}\text { Group A had more depressive symptoms } \\
\text { than group B. }\end{array}$ \\
\hline Avoid uncommon abbreviations & Group A had a faster 20MWT time than group B. & Group A walked faster than group B. \\
\hline Do not interpret the results & $\begin{array}{l}\text { Participants that received the intervention improved } \\
\text { more than participants that received a placebo, which } \\
\text { demonstrates the superiority of the intervention. }\end{array}$ & $\begin{array}{l}\text { Participants that received the intervention } \\
\text { improved more than participants that } \\
\text { received a placebo. }\end{array}$ \\
\hline
\end{tabular}


Table 2 A Suboptimal Table Design: Gait and Ankle Instability Outcomes of Participants Before and After Herbal Supplement Consumption

\begin{tabular}{lccc}
\hline Group & Distance Walked $(\mathbf{m})$ & $\begin{array}{c}\text { Cadence } \\
\text { (steps/min) }\end{array}$ & Number of Episodes of Giving Way \\
\hline CAI preherbal supplement & $50.3 \pm 10.6$ & $65.2 \pm 5.0$ & $6.0 \pm 2.0$ \\
CAI postherbal supplement & $75.6 \pm 9.2^{* \dagger}$ & $70.0 \pm 5.0$ & $3.5 \pm 2.0$ \\
Control preherbal supplement & $72.9 \pm 6.8$ & $68.3 \pm 5.0$ & $0.0 \pm 0.0$ \\
Control postherbal supplement & $74.6 \pm 7.0$ & $68.3 \pm 5.0$ & $0.0 \pm 0.0$ \\
\hline
\end{tabular}

$*$ Significantly different from baseline $(p<.05)$. $†$ Significantly different from control $(p<.05)$.

Table 3 An Optimal Table Design: Gait and Ankle Instability Outcomes of Participants Before and After Herbal Supplement Consumption

\begin{tabular}{|c|c|c|c|c|}
\hline \multirow[b]{2}{*}{ Outcome Measure } & \multicolumn{2}{|c|}{ Chronic Ankle Instability } & \multicolumn{2}{|c|}{ Control } \\
\hline & $\begin{array}{l}\text { Preherbal } \\
\text { supplement }\end{array}$ & $\begin{array}{l}\text { Postherbal } \\
\text { supplement }\end{array}$ & $\begin{array}{l}\text { Preherbal } \\
\text { supplement }\end{array}$ & $\begin{array}{l}\text { Postherbal } \\
\text { supplement }\end{array}$ \\
\hline Distance walked (m) & $50.3 \pm 10.6$ & $75.6 \pm 9.2 * \dagger$ & $72.9 \pm 6.8$ & $74.6 \pm 7.0$ \\
\hline Cadence (steps/min) & $65.2 \pm 5.0$ & $70.0 \pm 5.0$ & $68.3 \pm 5.0$ & $68.3 \pm 5.0$ \\
\hline Number of episodes of giving way & $6.0 \pm 2.0$ & $3.5 \pm 2.0$ & $0.0 \pm 0.0$ & $0.0 \pm 0.0$ \\
\hline
\end{tabular}

$*$ Significantly different from baseline $(p=.001)$. $†$ Significantly different from control $(p=.03)$.

\section{Making a Figure Worth 1,000 Words}

Figures are another great way to efficiently convey information or offer a more nuanced understanding of the raw data. The first figure can sometimes be used to help convey how the sample size was selected. For example, how did we get from screening 100 people down to the 10 people who completed the study? We commonly see this type of figure with clinical trials and systematic reviews/ meta-analyses, but they are applicable to case series, case-control, and cohort studies as well. Subsequent figures can communicate group differences or changes over time but also offer a more nuanced picture of the raw data than we could describe in the text. We encourage readers to abandon simple plots that rely solely on means and standard deviations and instead suggest that they provide readers with richer plots such as box plots or violin plots. Jay Hertel published a great article that we recommend for examples of valuable methods to display results, which balance the goals of providing simple-tointerpret results with a better picture of how each participant contributed to the analyses. ${ }^{4}$ An advantage of these figures is that it also avoids redundancy with the text or tables by providing data from each participant. Another example of these informative figures that IJATT editors often request is the Bland-Altman plot, which provides a more nuanced understanding of how a new measurement compares to a standard measurement (e.g., mobile phone app vs. inclinometer or goniometer). The plot shows the data from each participant with an $\mathrm{x}$-axis representing the average of the two measurements and the $y$-axis illustrating the difference between the two measurements. This graph also includes a $95 \%$ confidence interval for the amount of agreement and helps a reader easily detect a bias in a measurement (e.g., overall difference between new vs. old measure, or a difference between measurements that gets worse/better across the range of data). Besides having rich figures, it is also important to keep in mind that the figures need to be able to stand alone from the paper. Like a table, the title should tell a reader what is in the figure or what is the key take-home message. Furthermore, the figure legend needs to define all the abbreviations, symbols, and colors (but, keep in mind that using color often does not translate well in journals that print hard copies in black and white).

\section{Concluding Remarks}

The results section is often the shortest section of a paper, but it is likely a place where we lose many readers. If we want the results section to achieve our goal of offering evidence in a logical order to convince readers that our claims in the discussion and conclusions are real, it is pivotal that we write the results with our target audience in mind. This may require designing well-thought-out tables and figures and leveraging those resources to provide a simpler text that clearly communicates the key results that are needed to support key takehome messages. Failure to deliver a clear message throughout a paper could diminish the impact of a paper, which undervalues the time researchers and participants spent contributing to new findings. Hence, we have an obligation to our participants and study team to ensure we can maximize the chance that our study will help achieve the goal of improving human health.

\section{References}

1. Concato J, Hartigan JA. P values: from suggestion to superstition. J Investig Med. 2016;64:1166-1171. PubMed ID: 27489256 doi:10. 1136/jim-2016-000206

2. Wilkerson GB, Denegar CR. A growing consensus for change in interpretation of clinical research evidence. J Athl Train. 2018;53(3): 320-326. PubMed ID: 29624454 doi:10.4085/1062-6050-8-17

3. Day RA. How to Write \& Publish a Scientific Paper. 4th ed. Phoenix, AZ: Oryx Press; 1994.

4. Hertel J. A picture tells 1000 words (but most results graphs do not): 21 alternatives to simple bar and line graphs. Clin Sports Med. 2018;37(3): 441-462. PubMed ID: 29903385 doi:10.1016/j.csm.2018.04.001 